\title{
Treatment of patch infection after carotid endarterectomy: a systematic review
}

\author{
Tim M. Wikkeling, Senne A. van Gijssel, Maarten J. van der Laan, Clark J. Zeebregts, Ben R. Saleem \\ Division of Vascular Surgery, Department of Surgery, University Medical Center Groningen, University of Groningen, Groningen, The Netherlands \\ Contributions: (I) Conception and design: All authors; (II) Administrative support: None; (III) Provision of study materials or patients: None; (IV) \\ Collection and assembly of data: TM Wikkeling, SA van Gijssel; (V) Data analysis and interpretation: TM Wikkeling, SA van Gijssel, BR Saleem, MJ \\ van der Laan; (VI) Manuscript writing: All authors; (VII) Final approval of manuscript: All authors. \\ Correspondence to: Ben R. Saleem, MD, PhD, FEBVS. Division of Vascular Surgery, Department of Surgery, University Medical Center Groningen, \\ University of Groningen, P.O. Box 30.001, 9700 RB Groningen, The Netherlands. Email: b.r.saleem@umcg.nl.
}

\begin{abstract}
Carotid endarterectomy (CEA) with patch angioplasty is a widely used method for treating carotid artery stenosis. Patch infections are extremely rare, but the consequences may be serious. The current gold standard for treatment is patch excision and reconstruction with autologous material. However, no consensus has been reached and other options may be valuable as well in certain cases. The objective of this study was to evaluate the various treatment options for carotid patch infection after CEA with patch angioplasty on the basis of their outcomes (reinfection, ischemic stroke, and infection-related mortality). This systematic review was conducted in accordance with the PRISMA statement. The electronic bibliographic databases PubMed, Cochrane, and EMBASE were searched. Case series and case reports were included. Studies in languages other than English were excluded. Patients who developed a postoperative patch infection of CEA with patch angioplasty were included. Angioplasty could be performed with any type of patch. Patch infection needed to be confirmed by clinical presentation in combination with imaging, culture, or during the operation. The primary outcome measures were reinfection, ischemic stroke, and infection-related mortality. Eleven retrospective case series, two prospective case series, and seventeen case reports were included. The study size was 165 patients (mean age 69.7 years, M/F ratio 1.75:1). One hundred and seventy-one patches developed a patch infection after CEA with patch angioplasty and needed treatment. Treatment strategies included conservative treatment (14.0\%), endovascular treatment (4.7\%), and open surgery (81.4\%). Mean follow-up was 34.8 months and extended up to 180 months. Reinfection rate was $4.7 \%$, ischemic stroke rate $5.8 \%$, and infection-related mortality rate $2.3 \%$. No statistical comparison between treatment options could be performed, because of the heterogeneity of the included studies. Autologous material should be the primary choice of treatment if patch infection is diagnosed after CEA with patch angioplasty. In emergency situations, endovascular treatment, carotid ligation, or abscess drainage could be considered. Endovascular treatment and abscess drainage are temporary solutions. After the patient has recovered sufficiently, a more durable treatment i.e., open surgery is advised. Endo vacuum assisted closure (EndoVAC) seems to be promising. Further research is needed to determine the applicability of each treatment option.
\end{abstract}

Keywords: Carotid stenosis; carotid endarterectomy (CEA); angioplasty; patch infection

Submitted Nov 16, 2020. Accepted for publication Feb 26, 2021.

doi: $10.21037 /$ atm-20-7531

View this article at: http://dx.doi.org/10.21037/atm-20-7531 


\section{Introduction}

Carotid endarterectomy (CEA) and closure with patch angioplasty is a widely used method for treating carotid artery stenosis. Patch angioplasty is preferred over primary closure because of lower rates of postoperative restenosis, ischemic stroke, and infection-related mortality (1-3). Complications related to patch angioplasty are extremely rare, but patch infections do occur and range between $0.5-$ $1.0 \%$ (4). However, the incidence might be underestimated due to a lack of recognition and underreporting of the condition (5). Nevertheless, the consequences of a patch infection are serious.

It is estimated that $90 \%$ of patch infection are caused by Staphylococci and Streptococci species (4). The clinical presentation depends on the time of onset. Early presentation ( $\leq 4$ months) frequently consists of abscess formation, neck mass, hemorrhage, and patch rupture, while pseudoaneurysm and chronic sinus discharge are more likely signs of late presentation ( $>4$ months) $(4,5)$.

The gold standard for diagnosing patch infection after CEA is a positive culture of the patch material (5). However, clinical presentation and localization does not always allow preoperative culture to be obtained. Moreover, in some critical cases there is not enough time to wait for culture results and immediate intervention is required. Imaging could aid to establish the diagnosis of patch infection (4). In practice, the diagnosis of patch infection after CEA is based on the combination of clinical presentation, culture (if possible), and imaging.

There are various treatment options for patch infection, which may be divided into three main categories, including conservative treatment, endovascular treatment, and open surgery. Conservative treatment contains patch preservation combined with antimicrobial therapy and/ or abscess drainage. With endovascular treatment, the insertion of a covered stent takes place. Open surgery includes patch excision followed by patch reconstruction (vein/prosthesis), reconstruction with an interposition graft (vein/prosthesis), or carotid ligation (5). Antibiotics are given in all of these treatment options. Little is known about the outcomes of the different treatment options because of the low number of reported cases. A recent guideline issued by the European Society for Vascular Surgery (ESVS) based on a review of a total of 140 cases of supra aortic trunk patch, bypass, and stent infections recommended total removal of infected material following reconstruction with autologous material (5).
A systematic review was performed of treatment strategies of patch infection after CEA with patch angioplasty.

\section{Methods}

This systematic review was conducted in accordance with the Preferred Reporting Items for Systematic Reviews and Meta-Analysis (PRISMA) statement (6). We present the following article in accordance with the PRISMA 2020 checklist (available at http://dx.doi.org/10.21037/atm-207531) (6).

\section{Study objective}

The study objective was to evaluate the treatment options of carotid patch infection after CEA with patch angioplasty on the basis of their outcomes. The primary outcome measures were reinfection, ischemic stroke, and infection-related mortality. A systematic review was performed to identify all the types of interventions and their related outcomes.

\section{Data sources and search strategy}

A systematic search was performed by two authors (TM Wikkeling, SA van Gijssel) on July 5, 2020. The electronic bibliographic databases PubMed, Cochrane, and EMBASE were searched for Medical Subject Headings (MESH) terms and free text. In the search strategy, the terms 'CEA, patch, infection, and treatment' were combined and corresponding search terms were also included. The search term combinations were adapted to the peculiarities of each of the databases to be searched. Studies in languages other than English were excluded. There were no restrictions concerning the time period of publication applied. The search string for each database is listed in Appendix 1.

\section{Study selection}

Before running the search, the protocol was registered in the International prospective register of systematic reviews (PROSPERO, registration number CRD42020197142). The studies obtained from the search were screened for eligibility. The included study participants were patients who received treatment after developing a patch infection after CEA with patch angioplasty. Patients who underwent CEA with patch angioplasty and concomitant other operation(s) were also included. Patch infection needed to 
be confirmed by clinical presentation in combination with imaging, culture, or during the operation. Angioplasty could be performed with any type of patch. Information on postoperative course in terms of condition, complications, or mortality needed to be present. Included study designs were randomized controlled trials, observational studies, and descriptive studies. Animal research studies, opinions, and congress abstracts were excluded. Duplicates and overlapping databases were removed electronically with the citation management software Mendeley version 1.19.4 (Elsevier, London, UK). Subsequently, the remaining duplicates were removed manually.

The study selection was done by two review authors (TM Wikkeling, SA van Gijssel), who were going independently through the process to identify studies that potentially met the inclusion criteria outlined above. The initial selection was performed by screening the titles and/or abstracts with the review software Rayyan QCRI (Qatar Computing Research Institute, Doha, Qatar). The remaining articles were retrieved in full text and again assessed for eligibility. If the full-text version was not available, the study was excluded. Any discrepancies about the eligibility of particular studies were solved by requesting the full text versions in the first place. In case of disagreement at the eligibility of full text versions, consensus was reached through discussion with a third review author (BR Saleem).

\section{Data extraction}

Data extraction from included studies was performed by two review authors (TM Wikkeling, SA van Gijssel). The data extraction was done independently and cross-checked. In case of any discrepancies, consensus was gained by discussion with a third review author (BR Saleem). The collected study characteristics were publication year, study size, age, sex, patch material and number of patches during the index procedure, diagnostics (symptoms with time of onset, imaging, and causative organisms), treatment, and outcomes (follow-up, reinfection, ischemic stroke, and infection-related mortality).

\section{Assessment of study quality}

Two review authors (TM Wikkeling, SA van Gijssel) performed the quality assessment of the included studies. The quality assessments were carried out by the use of quality assessment tools. The National Institutes of Health (NIH) Quality Assessment Tool for Case Series Studies was used for the quality assessment of case series (7). The case series were classified as 'good', 'fair', or 'poor' based on the study objective, study population, consecutiveness of cases, compatibility, intervention description, outcome measures, follow-up length, statistical methods, and description of the results. The quality assessment of case reports was done by using the Joanna Briggs Institute (JBI) Critical Appraisal Checklist for Case Reports (8). The case reports were classified as 'good', 'fair', or 'poor' based on the patient's demographic characteristics, patient's history, current clinical condition, diagnostics, treatment procedure, postintervention clinical condition, complications, and the case report lesson.

\section{Data synthesis and statistical analysis}

The Statistical Package for the Social Sciences version 25.0 (SPSS, IBM, Armonk, New York, USA) was used for data collection and processing. Descriptive statistics were performed to present the study characteristics. The presentation of data was in mean, median, range, ratios, or percentages. Because of the limited number of patients included in each intervention and the lack of follow-up data, no statistical comparison between treatment options could be performed.

\section{Results}

After removal of duplicates, the search strategy yielded 655 potential studies. Eventually, 30 studies did meet all the inclusion criteria and were used for data extraction, synthesis, and analysis as shown in Figure 1.

\section{Study quality}

The systematic review provided eleven retrospective case series, two prospective case series, and seventeen case reports. A study was defined as case series when it documented all relevant patients from their patient population in a given time period. If the study not provided a certain period of time or not included all relevant patients, the study was defined as case report. No randomized controlled trials or observational studies were identified. The quality assessment of the case series according to the NIH Quality Assessment Tool for Case Series Studies is shown in Table 1. Nine studies were defined as good and four as fair. The NIH score averaged 6.8 (5 to 8, SD 0.87). Table 2 shows the quality assessment of the case reports 


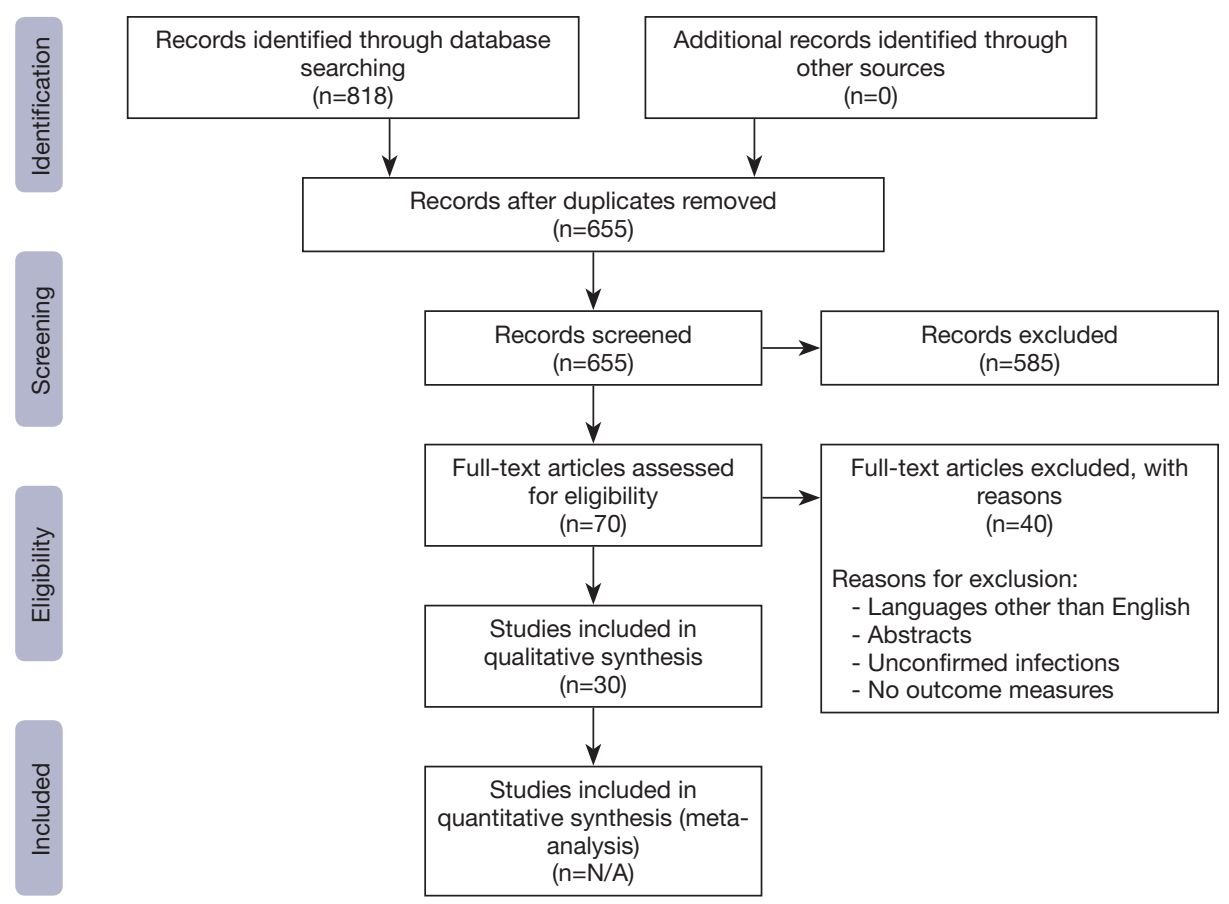

Figure 1 PRISMA Flow Diagram showing screening and selection of studies for systematic review (7).

according to the JBI Critical Appraisal Checklist for Case Reports. Nine studies were defined as good and eight as fair. The JBI score averaged 6.8 (5 to 8, SD 1.0).

\section{Study characteristics}

The study characteristics are summarized in Table 1 for the case series and in Table 2 for the case reports. The publication year of the studies varied from 1986 till 2019. The study size represented the number of patients treated for a patch infection after CEA with patch angioplasty. Three studies also included patients who underwent other operations, next to the patients who underwent CEA with angioplasty $(9,13,20)$. In three studies, patients with a bilateral CEA with angioplasty obtained a patch infection on both sides $(11,14,29)$. All patients were treated with antibiotics. Patch excision preceded repair and closure without patch, reconstruction with patch (vein or prosthesis), interposition with graft (vein, artery, prosthesis, or cadaveric homograft), carotid-carotid bypass, or carotid ligation. The veins and arteries used for patch or graft reconstruction were of autologous origin, with the exception of cadaveric homograft. In three patients, patch infection was not cured with the initial treatment $(11,22,34)$. The patients underwent another treatment, as shown in the Tables 1,2. Follow-up was done by clinical and/or by imaging assessment.

\section{Patient characteristics}

A total of 165 patients were included in this systematic review. The mean age of the included patients was 69.7 years, with a range of 47-91 years. The age was not provided in 33 patients. The sex ratio was 1.75:1 males/ females. The sex was not specified in 55 patients. 171 patches developed a postoperative patch infection of CEA and needed treatment. The material of the infected patches was Dacron $(52.0 \%)$, polytetrafluoroethylene (PTFE) (18.7\%), bovine pericardial (5.3\%), autologous vein (3.5\%), and unspecified $(20.5 \%)$. A total of 81 patients $(52.3 \%)$ developed an early infection ( $\leq 4$ months) and 74 patients $(47.7 \%)$ presented with a late infection ( $>4$ months). The time of onset was not mentioned in 17 patients. A total of 215 symptoms were reported and these were divided into early ( $\leq 4$ months) and late presentation ( $>4$ months). At early presentation, abscesses were frequently seen $(n=22$, 


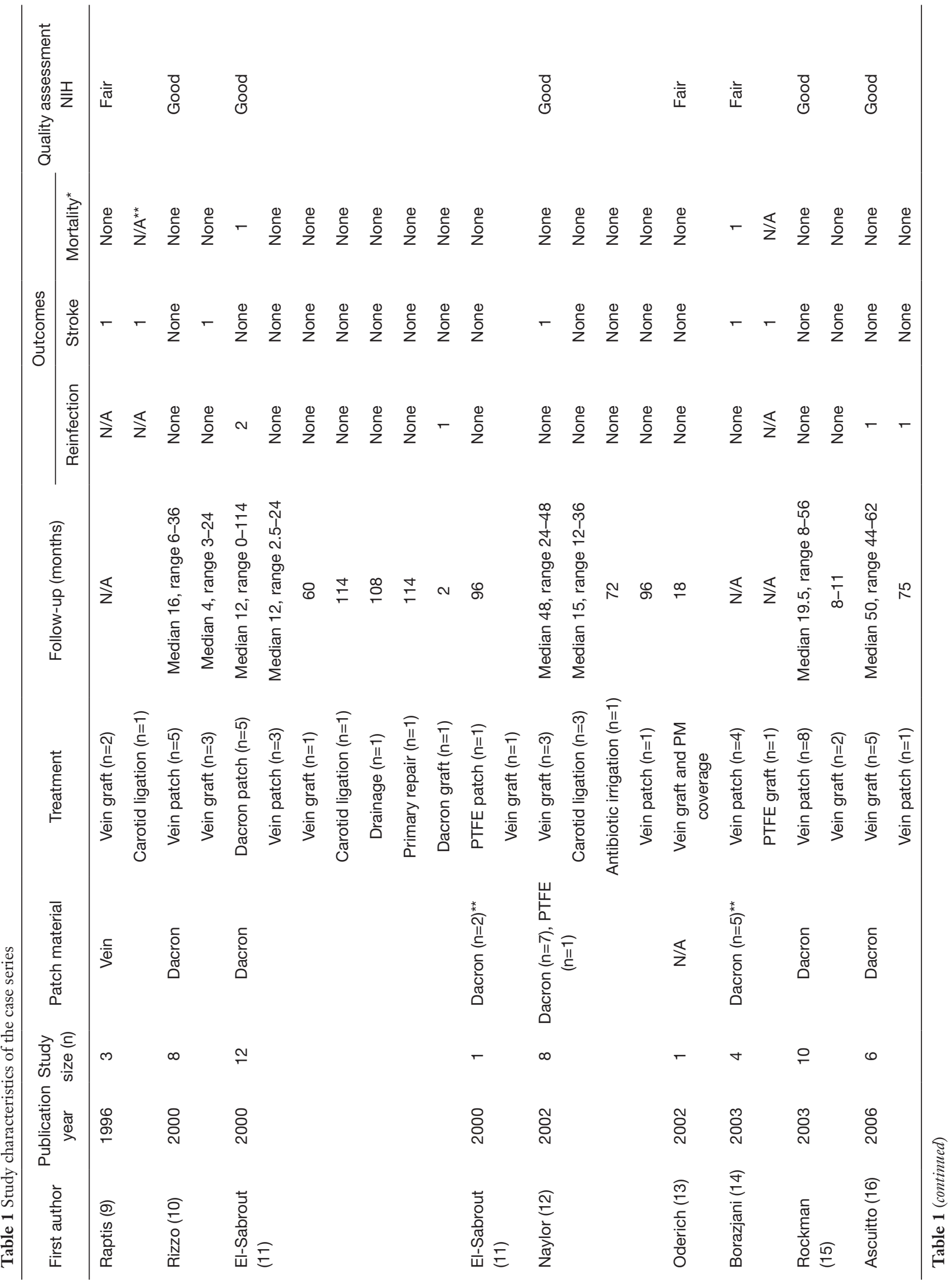




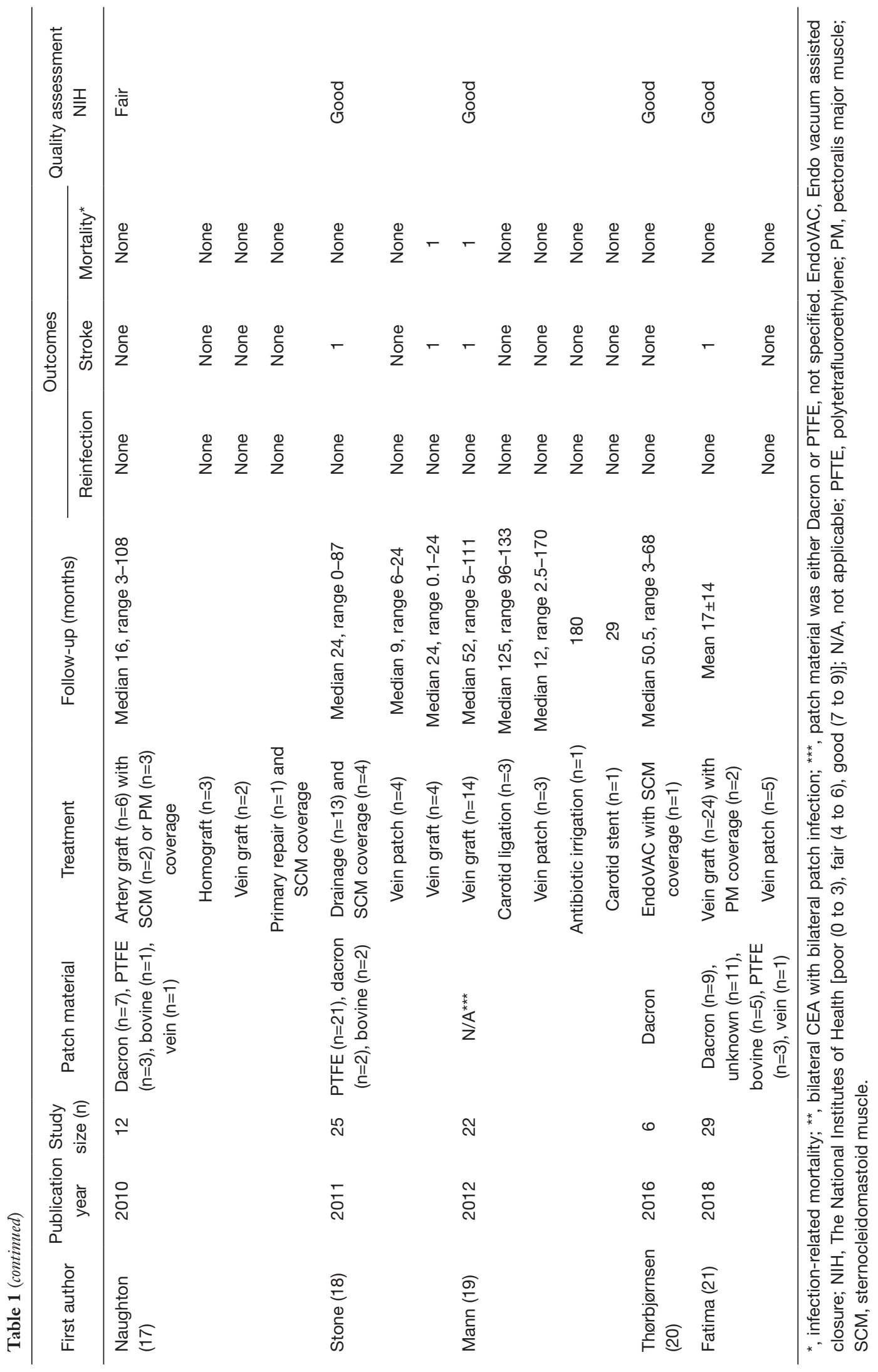




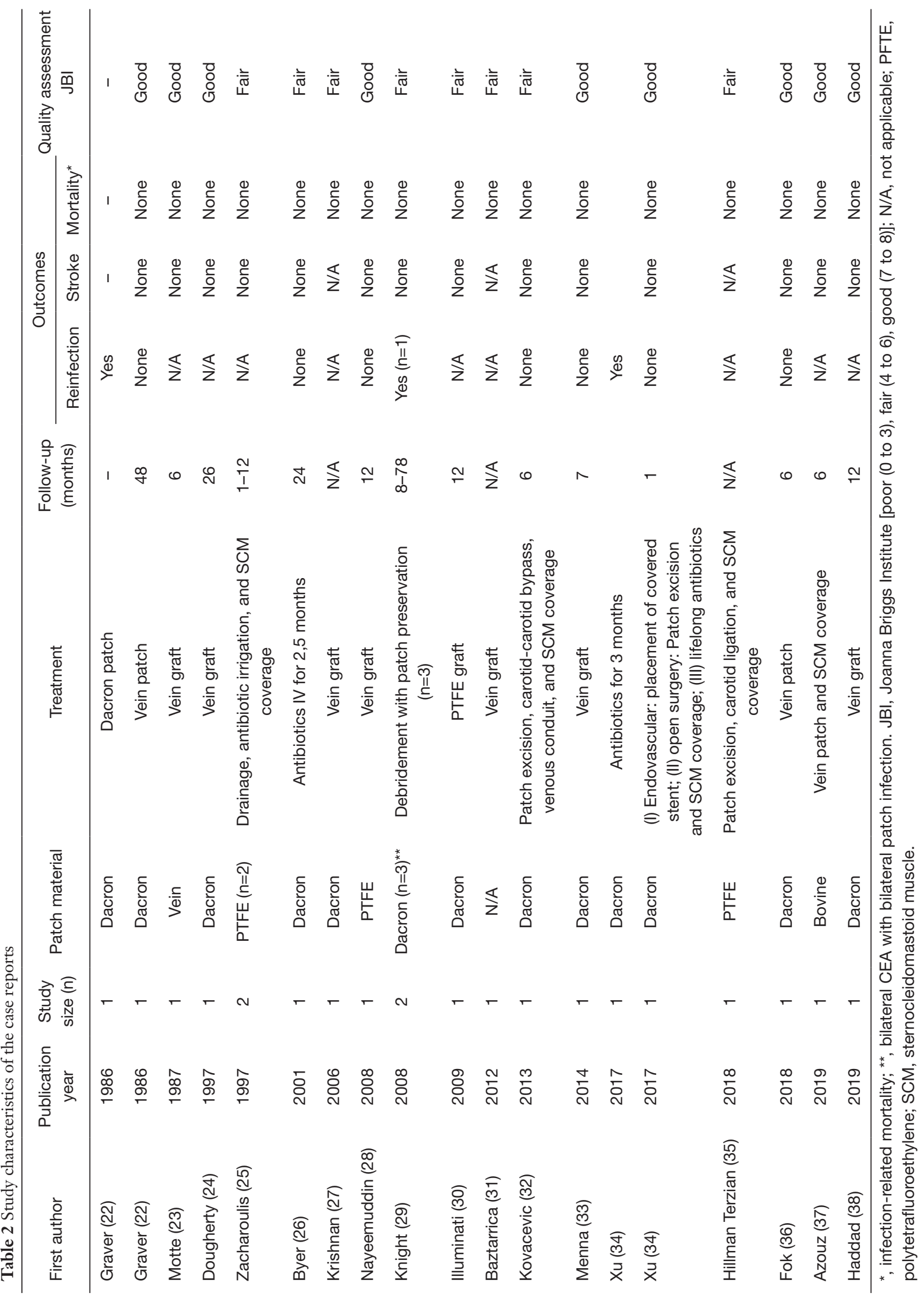


Table 3 Imaging tools used during diagnostic work-up

\begin{tabular}{lcc}
\hline Imaging & $\mathrm{N}$ & Percentage \\
\hline DU & 68 & $33.5 \%$ \\
CT and CTA & 56 & $27.6 \%$ \\
MRI and MRA & 17 & $8.4 \%$ \\
Angiography & 13 & $6.4 \%$ \\
Technetium-99m-labelled leukocyte & 4 & $2.0 \%$ \\
scan, PET/CT, SPECT/CT & & \\
Biopsy & 1 & $0.5 \%$ \\
No imaging done & 34 & $16.7 \%$ \\
N/A & 10 & $4.9 \%$ \\
Total & 203 & \\
\hline
\end{tabular}

CT, computed tomography; CTA, computed tomography angiography; DSA, digital subtraction angiography; DU, duplex ultrasound; MRA, magnetic resonance angiography; MRI, magnetic resonance imaging; N/A, not applicable; PET/CT, Positron emission computed tomography; SPECT/CT, single photon emission computed tomography.

Table 4 Causative organisms

\begin{tabular}{lcc}
\hline Organisms & $\mathrm{N}$ & Percentage \\
\hline Staphylococcus epidermidis & 22 & $12.5 \%$ \\
Staphylococcus aureus & 20 & $11.4 \%$ \\
MRSA/MRSE & 17 & $9.7 \%$ \\
Coagulase negative staphylococci & 12 & $6.8 \%$ \\
Streptococcus species & 16 & $9.1 \%$ \\
Other & 28 & $15.9 \%$ \\
Negative & 33 & $18.8 \%$ \\
No culture done & 10 & $5.7 \%$ \\
N/A & 18 & $10.2 \%$ \\
Total & 176 & \\
\hline
\end{tabular}

MRSA, methicillin-resistant staphylococcus aureus; MRSE, methicillin-resistant staphylococcus epidermis; N/A, not applicable.

$19.6 \%)$. Pseudoaneurysms ( $\mathrm{n}=35,34.0 \%)$ were mostly observed at late presentation. Sixty-nine reported symptoms could not be categorized into early or late presentation. Table 3 provides an overview of used diagnostics. As part of the diagnostic work-up, DU was performed the most $(33.5 \%)$. Table 4 shows the causative organisms of patch infection. Staphylococcus or Streptococcus species were mostly cultured in 105 patients (59.7\%). A total of 172 treatments were applied including $81.4 \%$ open surgery, $14.0 \%$ conservative treatment, and $4.7 \%$ endovascular treatment. Mean follow-up length was 34.8 months extending up to 180 months. The follow-up period was not reported in 24 patients. The reinfection rate was $4.7 \%$, the ischemic stroke rate $5.8 \%$, and the infection-related mortality rate $2.3 \%$.

\section{Outcomes of the different treatment options}

The treatment options were evaluated based on the primary outcomes (reinfection, ischemic stroke, and infectionrelated mortality).

\section{Conservative treatment}

A total of 24 patients $(14.0 \%)$ received conservative treatment with patch preservation. The primary outcomes were one reinfection $(4.2 \%)$, one ischemic stroke $(4.2 \%)$, and no infection-related mortality. Conservative treatment is further subdivided into intravenous antibiotics, antibiotic irrigation, abscess drainage, and abscess drainage with antibiotic irrigation. Two patients received intravenous antibiotics only (2.5-3 months) (26,34). Reinfection occurred in one patient $(50.0 \%)$. No ischemic stroke and infection-related mortality were reported. Antibiotic irrigation without abscess drainage was performed in two patients without postoperative complications $(12,19)$. In 18 patients, the abscess was drained without antibiotic irrigation. One ischemic stroke presented (5.6\%), with no reinfection or infection-related death $(11,18)$. Two patients were treated by abscess drainage with antibiotic irrigation without reinfection, ischemic stroke, and infection-related mortality (25).

\section{Endovascular treatment}

Endovascular treatment was performed in eight patients (4.7\%). Two patients were treated with a covered stent, one with patch preservation (19) and one with patch excision as a hybrid procedure (34). Six patients were treated in combination with the Endo vacuum assisted closure (EndoVAC) (20). The EndoVAC technique used consisted of (I) relining of the infected reconstruction with a stent graft; (II) surgical revision (without clamping the reconstruction); and (III) VAC therapy, to permit granulation and secondary delayed healing, and long-term antibiotic treatment. No 
reinfection, ischemic stroke, or infection-related mortality were reported for these endovascular treatments.

\section{Open surgery}

Open surgery was performed in 140 patients (81.4\%). Debridement with patch preservation was performed in three patients (29). In one of them a reinfection occurred $(33.3 \%)$. No ischemic stroke or infection-related death was reported. Two patients underwent patch excision with primary closure without postoperative complications $(11,17)$. In nine patients, the carotid artery was ligated after patch excision $(9,11,12,19,35)$. One of them suffered an ischemic stroke $(11.1 \%)$. No reinfection or infection-related death was reported. Patch excision followed by reconstruction with prosthetic patch, either Dacron or PTFE, was performed in seven patients $(11,22)$. There were three reinfections in this subgroup $(42.9 \%)$, one infection-related death $(14.3 \%)$, and no ischemic stroke. In 37 patients, reconstruction with an autologous vein patch after patch excision was performed $(10,12,14-16,18,19,21,22,36,37)$, resulting in one reinfection, one ischemic stroke, and one infectionrelated death (all 2.7\%). Patch excision followed by interposition with autologous vein was done in 69 patients (9-13,15-19,21,23,24,27,28,31,33,38). One reinfection $(1.4 \%)$, six ischemic strokes $(8.7 \%)$, and two infectionrelated deaths $(2.9 \%)$ were reported in this subgroup. Three patients underwent patch excision followed by interposition with prosthetic graft $(11,14,30)$. In this subgroup, one reinfection (33.3\%), one ischemic stroke $(33.3 \%)$, and no infection-related mortality occurred. In six patients, an arterial graft (superficial femoral artery) was used for reconstruction. In three patients, a cryopreserved artery homograft was used. One patient underwent a carotid-carotid bypass with venous conduit $(17,32)$. None of these treatments reported postoperative complications.

\section{Adjunctive treatment}

Additionally, sternocleidomastoid muscle (SCM) coverage was performed in 14 patients $(17,18,20,25,32,34,35,37)$. Six patients had a coverage with the pectoralis major muscle (PM) $(13,17,21)$. Muscle coverage was applied additional to several treatments, including drainage, endovascular approach, primary repair, carotid ligation, autologous vein patching, autologous artery and vein graft, and carotidcarotid bypass with venous conduit.

\section{Discussion}

This systematic review covers a time period of 33 years (1986 to 2019). During this period, imaging and treatment modalities underwent drastic improvements. Even new adjunctive therapies arose to the occasion. This systematic review demonstrates the variety in treatment options for patients with patch infection after carotid artery endarterectomy with patch angioplasty. The outcomes were reinfection $(4.7 \%)$, ischemic stroke $(5.8 \%)$, and infectionrelated mortality $(2.3 \%)$.

\section{Conservative treatment}

Fourteen percent of the reviewed cases were treated conservatively, with a reinfection rate of $4.2 \%$ and an ischemic stroke rate of $4.2 \%$. The ESVS guideline does not suggest conservative treatment because of possible major consequences for suture line rupture and vascular wall necrosis (5). According to Naylor, conservative treatment is used as a last resort and the existing documentation only outlines the surviving patients (4). Stone et al. suggested that there could be a role in limited surgical procedures combined with long-term antibiotics in selected patients and depending on the causative agent (18). Therefore, conservative treatment of postoperative patch infection of CEA is not recommended [unless the patient is unfit for surgery, e.g., advanced age with severe comorbidity or major stroke, level of evidence class IIb level C (5)].

\section{Endovascular treatment}

No reinfection, ischemic stroke, or infection-related mortality was seen in the endovascular treatment group. However, the number of patients within this group of treatments was small $(\mathrm{n}=8,4.7 \%)$. According to the ESVS guideline, endovascular treatment should be considered to gain control in life-threatening hemorrhage related to infection, level of evidence class IIb level C. The guideline also suggested the use of endovascular treatment in unstable patients as definitive therapy (5). Thorbjørnsen et al. reported six cases of Endo vacuum assisted closure (EndoVAC) (20). The evidence is limited as EndoVAC is only used in cases of severe comorbidities and adverse anatomy, level of evidence class IIb level C (5). The role of endovascular treatment of patch infection after CEA needs further exploration. 


\section{Open surgery}

In more than $80 \%$ of cases with an infected carotid patch, open surgery was performed. Debridement with patch preservation had a reinfection rate of $33.3 \%$. No postoperative complications occurred in patch excision with primary repair. Primary repair is not recommended due to the higher risk of stenosis in comparison with patch angioplasty (39). Carotid ligation with patch excision had an ischemic stroke rate of $11.1 \%$. The ESVS guideline and Naylor stated that carotid ligation could function as last resort in life threatening situation, if the infected reconstruction is already thrombosed without neurological symptoms or with an already completed cerebral infarct $(4,5)$. To conclude, the usage of carotid ligation is considered as a last resort. Patch excision and reconstruction with an autologous vein had low primary outcome rates for both patch (all 2.7\%) and interposition graft repair (reinfection $1.4 \%$, ischemic stroke $8.7 \%$, and infection-related mortality $2.9 \%$ ). The ESVS guideline recommends that autologous material is considered as the primary choice, level of evidence class I level C (5). Nonetheless, harvesting an autologous vein may have complications at the donor site. For instance, Naughton et al. reported groin seroma and Fatima et al. reported leg swelling and cutaneous nerve palsy $(17,21)$. Alternatively, Rockman et al. mentioned the advantages of prosthetic material as readily available, reliable, and technically easy to use (15). However, the results of this systematic review show that reconstruction with prosthetic material is associated with high outcome rates of reinfection, ischemic stroke, and infection-related mortality for both patch and graft. Prosthetic patch repair had a reinfection rate of $42.9 \%$ and an infection-related mortality rate of $14.3 \%$. Prosthetic interposition graft had a reinfection rate of $33.3 \%$ and an ischemic stroke rate of $33.3 \%$. This is also seen in the study conducted by Naylor (4). Therefore, the usage of autologous vein material over prosthetic material is preferred. Naughton et al. reported successful cases of patch excision and interposition with an artery graft or cryopreserved artery homograft (17). Nevertheless, these methods are rarely bail-outs because of the availability. Carotid-carotid bypass with venous conduit is a controversial treatment method. This procedure is not recommended because of the extensiveness and the involvement of the unaffected carotid.

\section{Adjunctive treatment}

Coverage of the wound with a SCM or PM flap could be used as adjunctive therapy (5). Zacharoulis et al. stated that the usage of well-vascularized tissue, such as SCM, improves healing time and minimizes infection, but caution concerning the blood supply of the muscle is essential to prevent ischemic damage (25).

\section{Antimicrobial therapy}

All patients within studies included in this systematic review were treated with antibiotics. Antimicrobial therapy was not specified because of missing data about the antibiotic type, dosage, formulation, and treatment duration in most cases. From the available information, an unequivocal treatment policy cannot be extrapolated as this depends on the causative organism. When patch infection is suspected, broad-spectrum antibiotics should be started. If culture reveals the causative organism, the spectrum can be narrowed down (5). According to the ESVS guideline, lifelong antimicrobial treatment is indicated for conservatively treated patients. Patients who underwent endovascular treatment should receive long-term antimicrobial therapy. For primary repair, a minimal of two weeks intravenous antibiotics followed by two to four weeks oral antibiotics is advised. A time period of four to six weeks of intensive antimicrobial therapy is indicated for patients treated with a patch or graft replacement (5).

\section{Limitations}

An inherent limitation of this review is the heterogeneity of the included studies. Several studies were incomplete, since they were missing data or did not have an adequate length of follow-up. The variability of the reported follow-up and outcomes made comparison of included studies impossible. Therefore, the extracted data was not appropriate for metaanalysis. Another important limitation is the difference in gold standard of the diagnosis. Many studies have an inconclusive confirmed diagnosis of patch infection. The ideal method of diagnosing patch infection is identifying the micro-organism by culture of the patch (5). Despite a present patch infection, a culture is not always positive and particularly low-grade infections remain undetected. Naylor stated that imaging may be helpful in diagnosing patch infection in more subtle situations (4). According to our results, duplex ultrasound (DU) was used most often (about $1 / 3$ of all imaging). With DU, pseudoaneurysms and gas or fluid collections can be detected and the patency of endarterectomized carotid artery can be checked. 
Another possibility is distinguishment between abscess and hematoma. DU may even be able to indicate infection at an early stage, by displaying carotid patch corrugation $(4,5)$. These characteristics make DU a good primary imaging screening modality, especially for superficial vascular grafts. Currently, to our knowledge, no data on the accuracy of DU in diagnosing vascular graft infection have been reported. As a second imaging modality, computed tomography angiography (CTA) is often used. CTA is able to show abscess formation, pseudoaneurysm, thrombosis, and perivascular stranding $(4,5)$. In case of emergency, CTA is the primary choice of diagnostics (4). The sensitivity and specificity of CTA to identify vascular graft or endograft infection are better than DU, being 0.67 (95\% CI: $0.57-$ 0.75 ) and 0.63 (95\% CI: 0.48-0.76), respectively (40). Magnetic resonance imaging (MRI), magnetic resonance angiography (MRA), 18F-fluoro-D-deoxyglucose positron emission tomography $\left({ }^{18} \mathrm{~F}-\mathrm{FDG}-\mathrm{PET}\right)$, and single photon emission computed tomography (SPECT/CT) in combination with technetium-99m-labeled leukocyte scan are less performed $(4,5)$. However, negative imaging does not necessarily rule out underlying infection (4). This made it difficult to determine if the patch was really infected or not. Because of the low incidence of patch infection, the existing literature lacks large studies with extensive numbers of patients. No studies with a control group were reported (inherent selection bias). This is necessary in order to be able to take major steps in the future. Publication bias arises from the tendency to only publish the cases with good results. Therefore, determination of the actual outcomes of treatment of patch infection is impeded (4). There is selection bias through the inclusion of English and published articles only.

In conclusion, if infection is diagnosed after CEA with patch angioplasty the patch should be removed and replaced by autologous material. Autologous material should be the primary choice of treatment. In emergency situations, endovascular treatment, carotid ligation, or abscess drainage could be considered. Endovascular treatment and abscess drainage are temporary solutions. After the patient has recovered sufficiently, a more durable treatment i.e., open surgery is advised. Endo vacuum assisted closure (EndoVAC) seems to be promising. Further research is needed to determine the applicability of each treatment option.

\section{Acknowledgments}

Funding: None.

\section{Footnote}

Provenance and Peer Review: This article was commissioned by the Guest Editor (Kosmas I. Paraskevas) for the series "Carotid Artery Stenosis and Stroke - Prevention and Treatment Part II" published in Annals of Translational Medicine. The article has undergone external peer review.

Reporting Checklist: The authors have completed the PRISMA reporting checklist. Available at http://dx.doi. org/10.21037/atm-20-7531

Conflicts of Interest: All authors have completed the ICMJE uniform disclosure form (available at http://dx.doi. org/10.21037/atm-20-7531). The series "Carotid Artery Stenosis and Stroke - Prevention and Treatment Part II" was commissioned by the editorial office without any funding or sponsorship. The authors have no other conflicts of interest to declare.

Ethical Statement: The authors are accountable for all aspects of the work in ensuring that questions related to the accuracy or integrity of any part of the work are appropriately investigated and resolved.

Open Access Statement: This is an Open Access article distributed in accordance with the Creative Commons Attribution-NonCommercial-NoDerivs 4.0 International License (CC BY-NC-ND 4.0), which permits the noncommercial replication and distribution of the article with the strict proviso that no changes or edits are made and the original work is properly cited (including links to both the formal publication through the relevant DOI and the license). See: https://creativecommons.org/licenses/by-nc-nd/4.0/.

\section{References}

1. Counsell CE, Salinas R, Naylor R, et al. A systematic review of the randomised trials of carotid patch angioplasty in carotid endarterectomy. Eur J Vasc Endovasc Surg 1997;13:345-54.

2. Bond R, Rerkasem K, Naylor AR, et al. Systematic review of randomized controlled trials of patch angioplasty versus primary closure and different types of patch materials during carotid endarterectomy. J Vasc Surg 2004;40:1126-35.

3. Rerkasem K, Rothwell PM. Systematic review of randomized controlled trials of patch angioplasty versus primary closure 
and different types of patch materials during carotid endarterectomy. Asian J Surg 2011;34:32-40.

4. Naylor R. Management of prosthetic patch infection after CEA. J Cardiovasc Surg (Torino) 2016;57:137-44.

5. Chakfé N, Diener H, Lejay A, et al. European Society for Vascular Surgery (ESVS) 2020 Clinical Practice Guidelines on the Management of Vascular Graft and Stent Graft Infections. Eur J Vasc Endovasc Surg 2020;59:339-84.

6. Moher D, Liberati A, Tetzlaff J, et al. The PRISMA Group (2009) Preferred Reporting Items for Systematic Reviews and Meta-Analyses: The PRISMA Statement. PLoS Med 2009;6:e1000097.

7. National Institute of Mental Health (2013). Study Quality Assessment Tools. Available online: https://www.nhlbi.nih. gov/health-topics/study-quality-assessment-tools

8. Aromataris E, Munn Z (Editors). JBI Manual for Evidence Synthesis. JBI, 2020. Available online: https:// synthesismanual.jbi.global

9. Raptis S, Baker SR. Infected false aneurysms of the carotid arteries after carotid endarterectomy. Eur J Vasc Endovasc Surg 1996;11:148-52.

10. Rizzo A, Hertzer NR, O'hara PJ, et al. Dacron carotid patch infection: a report of eight cases. J Vasc Surg 2000;32:602-6.

11. El-Sabrout R, Reul G, Cooley DA. Infected postcarotid endarterectomy pseudoaneurysms: retrospective review of a series. Ann Vasc Surg 2000;14:239-47.

12. Naylor AR, Payne D, London NJ, et al. Prosthetic patch infection after carotid endarterectomy. Eur J Vasc Endovasc Surg 2002;23:11-6.

13. Oderich GS, Panneton JM, Cherry KJ Jr, et al. Carotid artery reconstruction combined with myocutaneous flap coverage: a complex and durable rescue operation. Ann Vasc Surg 2002;16:579-85.

14. Borazjani BH, Wilson SE, Fujitani RM, et al. Postoperative complications of carotid patching: pseudoaneurysm and infection. Ann Vasc Surg 2003;17:156-61.

15. Rockman CB, Su WT, Domenig C, et al. Postoperative infection associated with polyester patch angioplasty after carotid endarterectomy. J Vasc Surg 2003;38:251-6.

16. Asciutto G, Geier B, Marpe B, et al. Dacron patch infection after carotid angioplasty. A report of 6 cases. Eur J Vasc Endovasc Surg 2007;33:55-7.

17. Naughton PA, Garcia-Toca M, Rodriguez HE, et al. Carotid artery reconstruction for infected carotid patches. Eur J Vasc Endovasc Surg 2010;40:492-8.

18. Stone PA, Srivastava M, Campbell JE, et al. A 10-year experience of infection following carotid endarterectomy with patch angioplasty. J Vasc Surg 2011;53:1473-7.

19. Mann CD, McCarthy M, Nasim A, et al. Management and outcome of prosthetic patch infection after carotid endarterectomy: a single-centre series and systematic review of the literature. Eur J Vasc Endovasc Surg 2012;44:20-6.

20. Thorbjørnsen K, Djavani Gidlund K, Björck M, et al. Long-term outcome after EndoVAC hybrid repair of infected vascular reconstructions. Eur J Vasc Endovasc Surg 2016;51:724-32.

21. Fatima J, Federico VP, Scali ST, et al. Management of patch infections after carotid endarterectomy and utility of femoral vein interposition bypass graft. J Vasc Surg 2019;69:1815-23.e1.

22. Graver LM, Mulcare RJ. Pseudoaneurysm after carotid endarterectomy. J Cardiovasc Surg (Torino) 1986;27:294-7.

23. Motte S, Wautrecht JC, Bellens B, et al. Infected false aneurysm following carotid endarterectomy with vein patch angioplasty. J Cardiovasc Surg (Torino) 1987;28:734-6.

24. Dougherty MJ, Calligaro KD, DeLaurentis DA. Infected false aneurysm after carotid - A case report and review of literature. Vasc Surg 1997;31:791-6.

25. Zacharoulis DC, Gupta SK, Seymour P, et al. Use of muscle flap to cover infections of the carotid artery after carotid endarterectomy. J Vasc Surg 1997;25:769-73.

26. Byer A, Keys RC, Panush D, et al. Late infection of a Dacron carotid endarterectomy patch--a case report. Vasc Surg 2001;35:469-72.

27. Krishnan S, Clowes AW. Dacron patch infection after carotid endarterectomy: case report and review of the literature. Ann Vasc Surg 2006;20:672-7.

28. Nayeemuddin M, Bhogal RH, Hickey N. Late presentation of an infected carotid patch as a superficial wound nodule. Surg Infect (Larchmt) 2008;9:395-7.

29. Knight BC, Tait WF. Dacron patch infection following carotid endarterectomy: a systematic review of the literature. Eur J Vasc Endovasc Surg 2009;37:140-8.

30. Illuminati G, Calio' FG, D'Urso A, et al. Management of carotid Dacron patch infection: a case report using median sternotomy for proximal common carotid artery control and in situ polytetrafluoroethylene grafting. Ann Vasc Surg 2009;23:786.e1-786.e5.

31. Baztarrica GP, Diplotti C, Reolon E, et al. Pseudoaneurysm of the carotid artery: Severe complication after carotid endarterectomy. Rev Argent Cardiol 2012;80:481-3. 
32. Kovacevic P, Velicki L, Ivanovic V, et al. Carotidcarotid bypass as an option in the treatment of infected pseudoaneurysm after prosthetic carotid replacement. Ann Vasc Surg 2013;27:239.e13-6.

33. Menna D, Ruggiero M, Speziale F. Infected pseudoaneurysm after carotid endarterectomy: mismatch between clinical presentation and origin. Ann Vasc Surg 2014;28:740.e17-9.

34. Xu JH, Altaf N, Tosenovsky P, et al. Management challenges of late presentation Dacron patch infection after carotid endarterectomy. BMJ Case Rep 2017;2017:bcr2017221541.

35. Hillman Terzian WT, Schadt S, Sheth SU. Right carotidcutaneous fistula and right carotid pseudoaneurysm formation secondary to a chronically infected polyethylene terephthalate patch. Int J Crit Illn Inj Sci 2018;8:48-51.
36. Fok KC, Chan YC, Law Y, et al. Septic carotid endarterectomy patch as a result of preoperative tooth extraction. Ann Vasc Surg 2018;50:299.e1-4.

37. Azouz V, Fahmy JN, Kornbau C, Petrinec D. Recurrent pseudoaneurysm after carotid endarterectomy. J Vasc Surg Cases Innov Tech 2019;5:128-31.

38. Haddad F, Wehbe MR, Hmedeh C, et al. Bilateral carotid patch infection occurring 12 years following endarterectomy. Ann Vasc Surg 2020;65:285.e11-5.

39. Huizing E, Vos CG, van den Akker PJ, et al. A systematic review of patch angioplasty versus primary closure for carotid endarterectomy. J Vasc Surg 2019;69:1962-74.e4.

40. Reinders Folmer EI, Von Meijenfeldt GCI, Van der Laan MJ, et al. Diagnosis imaging in vascular graft infection: a systematic review and meta-analysis. Eur J Vasc Endovasc Surg 2018;56:719-29.
Cite this article as: Wikkeling TM, van Gijssel SA, van der Laan MJ, Zeebregts CJ, Saleem BR. Treatment of patch infection after carotid endarterectomy: a systematic review. Ann Transl Med 2021;9(14):1213. doi: 10.21037/atm-20-7531 


\section{Search strings}

\section{PubMed}

(“Endarterectomy, Carotid"[Mesh] OR carotid endarterectom*[tiab] OR CEA[tiab] OR carotid patch angioplast*[tiab] OR carotid artery endarterectom*[tiab] OR carotid surger*[tiab])

AND

(patch*[tiab] OR Dacron*[tiab] OR vein*[tiab] OR material*[tiab] OR pericard*[tiab] OR Bovine*[tiab] OR vascular*[tiab] OR Hemashield*[tiab] OR expanded*[tiab] OR PFTE*[tiab] OR ePFTE*[tiab] OR prosthetic[tiab] OR synthetic[tiab])

AND

(“Infections"[Mesh] OR infect*[tiab] OR reinfect*[tiab])

AND

("therapy"[Subheading] OR "surgery"[Subheading] OR treatment*[tiab] OR management*[tiab] OR reconstruction*[tiab] OR therap*[tiab] OR intervention*[tiab] OR repair*[tiab] OR reintervention*[tiab] OR interposition*[tiab] OR debridement*[tiab] OR excision*[tiab] OR reoperat*[tiab] OR exclusion*[tiab] OR remov*[tiab] OR carotid ligation*[tiab] OR vein*[tiab] OR patch angioplast*[tiab] OR insert*[tiab] OR configuration*[tiab] OR antibiotic*[tiab])

\section{EMBASE}

('carotid endarterectomy'/exp OR 'carotid endarterectom*':ab,ti OR CEA:ab,ti OR 'carotid patch angioplast*':ab,ti OR 'carotid artery endarterectom*':ab,ti OR 'carotid surger”':ab,ti)

AND

(patch*:ab,ti OR Dacron*:ab,ti OR vein*:ab,ti OR material*:ab,ti OR pericard*:ab,ti OR Bovine*:ab,ti OR vascular*:ab,ti OR

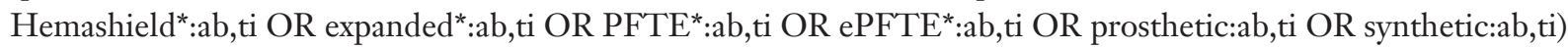

AND

('infection'/exp OR infect*:ab,ti OR reinfect*:ab,ti)

AND

('therapy'/exp OR 'surgery'/exp OR treatment":ab,ti OR management*:ab,ti OR reconstruction*:ab,ti OR therap*:ab,ti OR intervention*:ab,ti OR repair*:ab,ti OR reintervention*:ab,ti OR interposition*:ab,ti OR debridement*:ab,ti OR excision*:ab,ti OR reoperat*:ab,ti OR exclusion*:ab,ti OR remov*:ab,ti OR 'carotid ligation*':ab,ti OR vein*:ab,ti OR 'patch angioplast*':ab,ti OR insert*:ab,ti OR configuration*:ab,ti OR antibiotic*:ab,ti)

\section{Cochrane}

("carotid endarterectom*" OR CEA OR "carotid patch angioplast*" OR "carotid artery endarterectom*" OR "carotid surger*")

AND

(patch* OR Dacron* OR vein* OR material* OR pericard* OR Bovine* OR vascular* OR Hemashield* OR expanded* OR

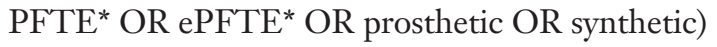

AND

(infect* OR reinfect*)

AND

(treatment* OR management* OR reconstruction* OR therap* OR intervention* OR repair* OR reintervention* OR interposition* OR debridement* OR excision* OR reoperat* OR exclusion* OR remov* OR "carotid ligation*" OR vein* OR "patch angioplast*" OR insert* OR configuration* OR antibiotic*) 